\title{
Study of chemical properties and skin tolerance activity of essential oils extracted from Rosmarinus Officinalis and Populusalba
}

\begin{abstract}
There are many plants which were the subject of recent research in the fields pharmaceutical, cosmetic and agroalimentary because of their chemical composition and their several therapeutic activities. Among these plants, the species of Rosmarinus officinalis (rosemary) and Populus alba (white poplar). The present study aims to examine the yield and chemical properties of essential oil of rosemary and white poplar. Then, the test of skin tolerance of essential oils was conducted by the measuring of primary irritation index. The results of chemical analysis reveal that the essential oils of the both plants are of acceptable quality. In other hand, the essential oil of Rosmarinus officinalis is non-irritating to the skin while the essential oil of Populus albais slightly irritating. This study is essential to ensure the safety of people in contact with substances in pharmaceutical applications.
\end{abstract}

Keywords: essential oils, populus alba, rosmarinus officinalis, irritation
Volume 2 Issue 4 - 2017

\author{
Belkhodja Hamza,' Meddah Boumediene, ${ }^{1,2}$ \\ Tir Touil Aicha' \\ 'Department of Microbiology Engineering and Health Safety, \\ Faculty SNV, University of Mascara, Algeria \\ ${ }^{2}$ Faculty of Pharmacy, University of Picardie, France
}

Correspondence: Belkhodja Hamza, Laboratory of Bioconversion, Microbiology Engineering and Health Safety, Faculty SNV, University of Mascara,Algeria,

E-mail hamzabelkhodja@yahoo.fr

Received: May 06, 2017 | Published: May 26, 2017

\section{Introduction}

For several years, the man who lives side by side with the plants is accustomed to consume for their medicinal and nutritional properties. Natural products are of great interest for the various sectors such as cosmetics, pharmaceuticals, food and industry. ${ }^{1}$ Currently, the World Health Organization (WHO) estimates that about $80 \%$ of people use traditional herbal preparations by lack of access to prescription drugs but also because the plants were able to demonstrate effectiveness. In addition, side effects induced by the drugs concerned users who turn to less aggressive care for the body. ${ }^{2}$ Indeed, these plants are often characterized by the biosynthesis of odorous molecules which are called"essential oils" (EO) long been known for their therapeutic activities in folk medicine. These bioactive molecules were used given their therapeutic properties in the treatment of several diseases affecting human health. ${ }^{3}$ Our work aims to examine the yield and chemical properties of essential oil of rosemary and white poplar. Then, the test of skin tolerance of both essential oils was conducted by the measuring of primary irritation index. This work was carried out at the laboratory of the University of Mascara, beginning the month of May 2014.

\section{Materials and methods}

\section{Plant material}

It was constituted of aerial parts (leaves and flowers) ${ }^{4}$ of the two plant species; Rosmarinus officinalis and Populusalba. The plants were collected and were identified in the Mascara region during the month of May 2014.

\section{The experimental animal}

The Wistar rats used in these experiments were provided by the laboratory of the University of Mascara. Animals were housed at the cage, with water and food Ad libitum, and the animal room temperature was kept at constant temperature of $20 \pm 1{ }^{\circ} \mathrm{C}$ on a 12 -hour light/12-hour dark cycle. Adequate measures were taken to minimize pain or discomfort of the animals, and all experimental procedures were performed in accordance with the ethical guidelines of the Organization for Economic Cooperation and Development (OECD).

\section{The physicochemical analyses}

a. Water content: The method used was the evaporative drying method. ${ }^{5}$

b. Ash content: The method used by the calcinations mineralization method. ${ }^{6,7}$

\section{Extraction of essential oils}

The extraction of essential oils from two plants (Rosmarinus officinalis and Populusalba) was conducted in the laboratories of the University of Mascara. The extraction of essential oils was carried out by hydro distillation in a Clevengerapparatus.100g of leaves and flowers of each plant was boiled. When the temperature stabilizes, we begin to collect the distillate in an Erlenmeyer. We add about $18 \mathrm{~g}$ of sodium chloride $(\mathrm{NaCl})$ to the distillate. We stir until dissolved, then it was placed in a separating funnel and we achieve three successive washes $(10,10,20 \mathrm{ml})$ of cyclohexane. After agitation, the organic phase was recovered. The product was dried with a little an hydroussodium sulfate. Then we make a concentration by rotary evaporator to obtain the essential oil. The essential oil obtained was stored at $+4^{\circ} \mathrm{C}$ after the calculation of the yield of extraction.

\section{Organoleptic characters}

According to AFNOR NF ISO 280: 1999, essential oils must respond to analytical characteristics that are established by international committees of experts. To know the quality of the EO of R. Officinalis (Rosemary) and P. Alba (white poplar), standard organoleptic tests like color and odor were performed.

a. Chemical indexes: ${ }^{8}$

b. pH: This measurement was taken using a $\mathrm{pH}$ meter. 
Acid index: (NF T 75 103, 1982) The acid expresses the number of milligrams of potassium hydroxide $(\mathrm{KOH})$ required to neutralize the free acids contained in one gram of essential oil. $2 \mathrm{~g}$ of essential oil was added to $5 \mathrm{ml}$ of ethanol $95 \%$ and 5 drops of phenolphthalein at $0.2 \%$. The solution was neutralized by the solution of $\mathrm{KOH}(0.1 \mathrm{~mol} /$ 1) until a pink color. The volume of the solution of $\mathrm{KOH}$ was denoted. The calculation of AI was given by the formula (1):

$$
\mathrm{AI}=5.61 \times \mathrm{V} / \mathrm{M}
$$

\subsection{1: Corresponds to $0.1 \mathrm{~mol} / \mathrm{L} \mathrm{KOH}$}

M: mass in grams of the essential oil

$\mathrm{V}$ : Volume in milliliters of ethanol solution of $\mathrm{KOH}(0.1 \mathrm{~mol} / \mathrm{l})$ used for titration.

Ester index: (NFT 75 104, 1982) The ester value is the number of milligrams of $\mathrm{KOH}$ needed to neutralize the free acids by hydrolysis of esters contained in one gram of essential oil. $2 \mathrm{~g}$ of essential oil was added to $25 \mathrm{ml}$ of ethanol solution of $\mathrm{KOH}(0.5 \mathrm{~mol} / 1)$. It adapts the condenser and placed the ball on the heating mantle and allowed to heat for one hour. Then, the solution was added to $20 \mathrm{ml}$ of distilled water and 5 drops of $0.2 \%$ PP. The excess of $\mathrm{KOH}$ solution was titrated with hydrochloric acid $0.5 \mathrm{~mol} / 1$. A blank test was carried out under the same conditions and with the same reagents. The calculation of EI was given by the formula (2):

$$
\mathrm{EI}=\left(28.05 \times\left(\mathrm{V}_{0}-\mathrm{V}_{1}\right) / \mathrm{M}\right)-\mathrm{IA}
$$

$28.05 \mathrm{~g} / 1$ : corresponding to $0.5 \mathrm{~mol} / \mathrm{L} \mathrm{KOH}$.

M: mass in grams of the test. blank.

$\mathrm{V}_{0}$ : Volume in $\mathrm{ml}$ of the $\mathrm{HCl}$ solution $(0.5 \mathrm{~mol} / 1)$ used for the

$\mathrm{V}_{1}$ : volume in $\mathrm{ml}$ of the $\mathrm{HCl}$ solution $(0.5 \mathrm{~mol} / \mathrm{l})$ used to determine the EI of the EO.

Skin tolerance test (PI): The skin tolerance test aims to predict from the knowledge of the toxicity on a living organism, the toxicological risk in humans. The index of primary skin irritation (PI) of our extracts was determined by official method published in the Official Journal of the French Republicon 21February 1982. The method was based on the observation of skin reactions caused by the applying of principle extract. $^{9}$

\section{Protocol}

The method was performedon06Wistarratsofbody weightbetween $280-350 \mathrm{~g}$. The back and sides were shaved to clear an area of approximately $5 \mathrm{~cm} \times 5 \mathrm{~cm}$; operate with caution so as to avoid irritation. Perform the right of the vertebral axis, using as sterile scalpel blade, three parallel scarifications over a length of about $2.5 \mathrm{~cm}$, spaced approximately $0.5 \mathrm{~cm}$. Apply the product on the skin (scarified areas and not scarified) at $0.5 \mathrm{~g}$. Place the protective compress. Remove the band age twenty-four hours after the applying. The readings were to collectery thema and edemain numerical scale of DRAIZE. To calculate the index of skin irritation(PI),we add the figures recorded for erythema and edema at each reading time ( 24 and 72 hours after application)on the six areas scarified and sixun scarified and then calculate the average of results observed to be classified according to the scale previously cited (3):

$\mathrm{PI}=(\text { edema }+ \text { erythema })_{\text {sidescarified }}+(\text { edema }+ \text { erythema })_{\text {intactside }} / 24$

With:24=number of rats (6)xnumber of tested areas (intact and scarified $=2$ ) $x$ number of type of irritation (erythema and edema=2).

The rating system used to generate the observed phenomena by calculating an index of primary skin irritation (PI) to classify the productin 4 categories:

a. Actionnon-irritating, PI less than 0.5: $\mathrm{PI}<0.5$

b. Action slightly irritating, PI between 0.5 to $2: 0.5<\mathrm{PI}<2$

c. Action moderately irritating, PI between 2 and $5: 2<\mathrm{PI}<5$

d. Severely irritating Action, PI between 5 and 8 : $5<\mathrm{PI}<8$

\section{Statistical analysis}

The values were expressed as mean \pm standard deviation (mean \pm $\mathrm{SD})$. The results of the different tests were analyzed by ANOVA single factor for multiple comparisons. The $\mathrm{P}$ values less than $0.05(\mathrm{p}<0.05)$ were considered statistically significant.

\section{Results and discussion}

\section{The physicochemical analyzes of the two plants}

A. Water and Ash content : Fresh plants were rich in water and contain from 60 to $80 \%$ water. ${ }^{10}$ We used the weight method for determining the water content in the leaves of our plants. It is the détermination of the mass loss by drying in an oven. The water content of our samples was of the order of $67.6 \pm 08 \%$ for rosemary and $73.4 \pm 05 \%$ for the white poplar (Figure 1). Albu and colleagues ${ }^{11}$ found water content of $40 \%$ in the fresh leaves of rosemary; this content differed remarkably with our results. We notice, however, that the two plants showed slightly difference in ash content $(3.2 \pm 05 \%$ for rosemary and $2.8 \pm 03 \%$ for the white poplar). Variations encountered in water content and ash content of our samples compared to some earlier work may be due to some environmental factors, age of the plant, the period of the growing season, or evengenetic factors. ${ }^{12}$
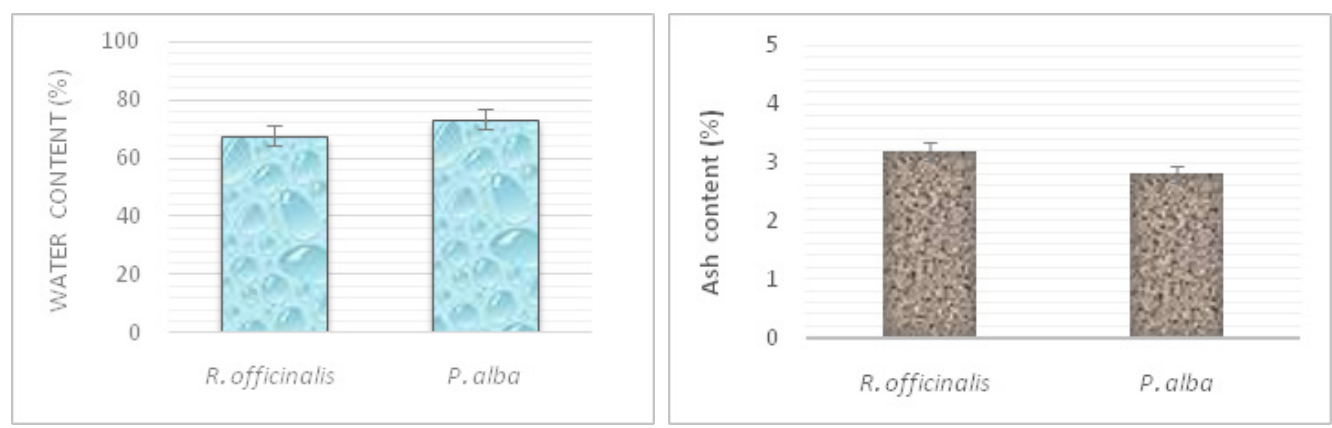

Figure I The water and ash content of the both plants. 


\section{Analysis of essential oils}

\section{Extraction yields}

The essential oil content, obtained from the aerial parts (leaves + flowers) was $1.29 \%$ for the Rosemary and $0.9 \%$ for white poplar. The yields of essential oils from two species were widely variable (Table 1). The yield of essential oil of Rosmarinus officinalis was higher than that quoted by Atik Bekkara et al. ${ }^{4}$ and those of Rouabeh ${ }^{13}$ where the quantities obtained by the set wo works arerespectively $0.8 \%$ and $0.9 \%$. Indeed, the extraction yield, as the quality of EO, are influenced by the type of soilon which the plantingis done, the material of the equipment used, the cleanliness of the equipment, the operating pressure, regularity the heating, the cooling of the distillate, method and distillation time. ${ }^{14}$

Table I Extraction yields

\begin{tabular}{llllll}
\hline Plant material & Mass of plant $(\mathbf{g})$ & Mass of extract $(\mathbf{g})$ & Aspect & Color & Y (\%) \\
\hline R. officinalis & 850 & II & oily & yellow & $1.29 \pm 0.3$ \\
P. alba & 850 & 7.8 & oily & pale yellow & $0.9 \pm 0.08$ \\
\hline
\end{tabular}

\section{Organoleptic characters}

It was through the organoleptic properties (appearance, color, smells) that it was possible to define that oil was of adequate quality. After comparison with the standard AFNOR method, the organoleptic properties of essential oils obtained from the two species suggest an essential oil of very good quality. For R.Officinalis, the EO has an oily liquid aspect, yellow with a powerful scent of rosemary flowers. While the species P.Alba, the EO has an oily liquid aspect, pale yellow.

\section{Chemical indexes of essential oils}

The values of chemical indexes were collected in Table 2 .
pH: pH measurement gave a value of 6.05 and 6.11 for the essential oil of $R$. Officinalis and P. Alba respectively.

Acid Index: The EO gave an AI of 0.421 for R. Officinalis and 0.530 for $P$. Alba (Table 2). In reality, a fresh essential oil contains very little free acid. ${ }^{17}$ The acid value must be as small as possible. Similarly, an acid number of less than 2 was an indicator of good quality of the oil.

Ester index: Our essential oils revealed an ester index of 28.1 for $R$. Officinalis and 18.3 for P. Alba. Over the ester index was high, the quality of HE will be better. ${ }^{18}$ According to these chemical properties, it appeared that our essential oils are of good quality.

Table 2 The chemical indexes of essential oil of R. officinalis and P. alba

\begin{tabular}{llllll}
\hline Properties & & EO of $\boldsymbol{R}$. officinalis & EO of $\boldsymbol{P}$. alba & \multicolumn{1}{c}{ Values of reference } & Reference \\
\hline & $\mathrm{pH}$ & 6.05 & $6.1 \mathrm{I}$ & 7 -Jun & \\
Chemical index & Acid Index & 0.421 & 0.53 & $0.5-2$ & 15 \\
& Ester index & 28.1 & 18.3 & - & -
\end{tabular}

\section{Test of skin tolerance $(\mathrm{PI})$}

The evaluation of skin irritation potential was essential to ensure the safety of people in contact with substances in pharmaceutical applications. ${ }^{19}$ Based on the results, the index of primary skin irritation of R.Officinalis essential oil was equal to 0.45 while that of the essential oil of $P$. Alba was equal to 0.66 . According to numerical Draize scale, it was concluded that the essential oil of R.Officinalis was non-irritating to the skin $(\mathrm{PI}<0.5)$, while the essential oil of P.Alba was slightly irritating $(0.5<\mathrm{PI}<2)$. From the results obtained, the observed phenomena were only erythema with a varying degree but a decrease was noted after 72 hours. While edema were totally absent in rats treated with the essential oil of $R$. Officinalis but for the essential oil of $P$. Alba, a slight appearance of edema during $24 \mathrm{~h}$ will be completely disappeared after 72 hours. The appearance of erythema in a few rats can due to penetration of the constituents of the oil in the epidermis. ${ }^{19}$ Thus, the appearance was quite noticeable on scarified flanks, which may be due to the scarification, which reaches the dermis. Some factors can affect the erythema phenomena such as rats friction between them during the period of accommodation and even hypersensitivity rats with oil constituents. ${ }^{20}$ Evaluation of skin irritation potential was essential to ensure the safety of people in contact with substances in pharmaceutical applications.

\section{Conclusion}

The results of chemical analysis reveal that the essential oils of the both plants were of acceptable quality. In other hand, the skin tolerance test on rats has allowed us to classify our essential oil of rosemary as non-irritating $(\mathrm{IP}=0.45)$, while that based on white poplar was seen as a product to slightly irritating action to the skin ( $\mathrm{IP}=0.66)$.

\section{Acknowledgements}

We express our sincere gratitude to all staff of the Directorate of Education of Mascara.

\section{Conflict of interest}

Author declares that there is no conflict of interest.

\section{References}

1. Bahorun T. Natural active substances: flora of Mauritius, a potential source of supply. Food and agricultural research council Mauritus. 1997:83-94.

2. Lhuillier A. Contribution to a phytochemical study of four Malagasy plantes: AgauriasalicifoliaHook.f ex Oliver, Agauriapolyphylla Baker (Ericaceae), Tambourissatrichophylla Baker (Monimiaceae) et Embeliaconcinna Baker (Myrsinaceae). PhD thesis. Toulouse, France; 2007.

3. Bensegueni A. Traditional ointments for the treatment of wounds and burns. Thesis state on veterinary science. University of Mentouri, Constantine, Algeria; 2007. 
4. Atik bekkara F, Bousmaha L, Talebbendiab SA, et al. Chemical composition of essential oil from Rosmarinus officinalis L pushing growing wild and cultivated in the Tlemcen region. Biology and Health. 2007;7:6-11.

5. Audigie C, Figarella J, Zonszaain F. Biochemical Manipulation. In: Doin, editor. Paris; 1978. p. 274.

6. Pinta M, Bourdou B, Rousselet F. Atomic absorption spectrophotometry. In: Masson, Arston, editor. Paris; 1980. p. 478.

7. AOAC. Officials methods of analysis. 11th ed. USA; William Horvi Washington: 1980

8. AFNOR. The French standards essential oils. AFNOR. 1992. p. 57.

9. Cohen Y, Pradeau D. Evaluation of skin tolerance in vivo, practical analysis of drugs. International medical editions. MOCLP. 1992. p. 7.

10. Paris R, Moyse H. Materia Medica precise: accurate collection of pharmacy. Paris; Masson: 1965. p . 412.

11. Albu S, Joyce E, Paniwnyk L, et al. Potential for the use of ultrasound in the extraction of antioxidants from Rosmarinus officinalis for the food and pharmaceutical industry. Ultrason Sonochem. 2004;11(3-4):261-265.

12. Laurent L. Mineral elements: analysis and control techniques in the food industry. 1991;4:78-98.
13. Rouabah Y. Contribution to a quantitative study of essential oils from two spieces: Globulariaalypum L. and Rosmarinus officinalis L. thesis, University of Batna, Algeria; 2010.

14. Brulé CH, Pecout W. The ylang-ylang: a subtle scent. UK; Grasse: 1995.

15. ISO 1342. Oil of rosemary (Rosmarinus officinalis L). ISO, Switzerland; 2000 .

16. ISO 709: 2001. Essential oils. Determination of acid index. NFT. 1994. p. $75-104$.

17. Dumortier D. Contribution to the improvement of the quality of the essential oil of ylang-ylang (Canangaodorata) of Comoros, University Faculty of Agronomic Sciences of Gembloux (Belgium); 2006.

18. Fauconnier ML. Ylang-ylang essential oil: its quality record and its distillation follow-up-Presentation for the GIE Spices House of Comoros. 2006.

19. Goossens A, Lepoittevin JP. Contact allergy to cosmetics and perfume components: new clinic, chemical and diagnostic aspects. French Journal of Allergy and Clinical Immunology. 2003;43(5):294-300.

20. Lis-Balchin M. Aromatherapy science: A guide for healthcare professionals. UK; Pharmaceutical Press: 2005. p. 195-201. 